\title{
¿Cómo se aplica la ingeniería del conocimiento en la investigación de mercados? Estudio de caso
}

\author{
How knowledge engineering is applied in market reach? Study of case \\ Vanessa Rodríguez-Lora ${ }^{*} \quad$ Alejandro Valencia Arias ${ }^{1}$ \\ Recibido 19 de enero de 2017, aceptado 26 de junio de 2017 \\ Received: January 19, 2017 Accepted: June 26, 2017
}

\begin{abstract}
RESUMEN
La ingeniería del conocimiento es un área de la computación empleada en las organizaciones como una estrategia para conservar el conocimiento clave y emplearlo de manera estratégica. Este artículo muestra como la investigación de mercados logra emplear la ingeniería de conocimiento como una herramienta fundamental en su búsqueda de información útil y altamente relevante que le permita identificar qué piensan sus clientes y poder orientar efectivamente sus esfuerzos.

Para ello, en este artículo se caracteriza el caso de una investigación en modelos de aceptación tecnológica del mobile learning al interior del Instituto Tecnológico Metropolitano, en el que por medio de un estudio de investigación de mercados usando herramientas de ingeniería de conocimiento, se identificaron los principales factores de aceptación del mobile learning en estudiantes universitarios, estos factores fueron: la autonomía en el aprendizaje, autoeficacia percibida, facilidad de uso percibida, preparación del instructor y utilidad percibida.

Entre los principales aportes de la investigación se encuentra mostrar cómo las técnicas y herramientas de la ingeniería del conocimiento van más allá del campo de la inteligencia artificial y se han convertido hoy en día en un mecanismo que permite adquirir el conocimiento de los participantes de un proyecto de conocimiento. El uso adecuado de estas técnicas ayuda a los proyectos en el logro de mejores resultados identificando el tipo de información requerida y quién es el dueño de esta para aplicar el instrumento adecuado para su obtención.
\end{abstract}

Palabras clave: Ingeniería del conocimiento; investigación de mercados; información, adquisición de conocimientos; representación de conocimientos.

\begin{abstract}
Knowledge engineering is an area of computing used in organizations as a strategy to conserve essential knowledge and use it strategically. This article shows how market research manages to use knowledge engineering as a fundamental tool in its search for useful and highly relevant information that allows identifying what its clients think, and expertly guide their efforts.
\end{abstract}

To this end, this article characterizes the case of research on technology acceptance models of mobile learning within the Metropolitan Technological Institute. Through a Market Research study using Knowledge Engineering tools, the Main factors of acceptance of mobile learning in university students,

\footnotetext{
1 Departamento de Ciencias Administrativas. Instituto Tecnológico Metropolitano -ITM-. A.A 54959. Medellín, Colombia. E-mail: vanessarodriguez@itm.edu.co; jhoanyvalencia@itm.edu.co

* Autor de correspondencia.
} 
these factors were: autonomy in learning, perceived self-efficacy, perceived easiness of use, instructor training and perceived usefulness.

Among the main contributions of the research is to show how the techniques and tools of Knowledge Engineering go beyond the field of artificial intelligence and have become nowadays a mechanism that allows acquiring the expertise of the participants of a knowledge project. The proper use of these techniques helps the projects to achieve better results by identifying the owner and type of information required, to apply the appropriate instrument for obtaining it.

Keywords: Knowledge engineering, market research; information, knowledge acquisition; knowledge representation.

\section{INTRODUCCIÓN}

La ingeniería del conocimiento es una disciplina derivada de la inteligencia artificial que ha venido usándose ampliamente en otras áreas de la administración y del mercadeo para que, por medio de una serie de técnicas, sea posible recopilar el conocimiento que tienen los clientes y usuarios de un servicio. Estas técnicas están clasificadas de acuerdo con el proceso que apoyan dentro de la ingeniería del conocimiento, así hay técnicas para hacer adquisición de conocimiento, otras para hacer representación del conocimiento y otras para realizar su manipulación [1]. Identificar las técnicas más apropiadas para un caso particular, es una de las grandes fortalezas que debe hacer quien desarrolla proyectos de conocimiento.

En este artículo muestra como dentro de un proceso de investigación de mercados se usan técnicas relacionadas con los procesos de adquisición y representación del conocimiento, como herramientas útiles para el ingeniero del conocimiento, en el caso particular de este artículo, un investigador de mercados, con el fin de facilitarles su labor.

Para ello, en los primeros apartes se presenta un marco conceptual que describe lo qué es la ingeniería del conocimiento y como esta puede usarse dentro de la investigación de mercados. Posteriormente se presentan las herramientas empleadas en un proyecto en particular de investigación de mercados dentro del proyecto de investigación que busca identificar los factores de aceptación del mobile learning entre los estudiantes del programa de administración tecnológica del Instituto Tecnológico Metropolitano. Por último, se presentan las conclusiones y las referencias bibliográficas utilizadas.

\section{ANÁLISIS TEÓRICO}

\section{¿Qué es la ingeniería del conocimiento?}

El conocimiento se entiende principalmente como un recurso, tanto de entrada como de salida para ciertas actividades, aunque se amplía su definición, ya que el concepto de conocimiento es discutido en varias disciplinas, lo que hace que su definición varíe [1]. Al respecto se plantea que usualmente, el conocimiento es confundido con información, pero esta es particularmente definida como una colección de datos relacionados entre sí, que son de suma importancia para la toma de decisiones en los diferentes ámbitos, es decir, puede decirse que el conocimiento es mucho más difícil de transmitir, de interpretar, de gestionar y de entender que la información en sí [2].

Otra de las definiciones más aceptadas del conocimiento, plantea que este es la justificación de las creencias y valores personales del "conocedor" o persona que posee el conocimiento, y este es fundamental para la generación y estructuración de este conocimiento [3]. Además, el conocimiento es dinámico, ya que permite a los interesados aprender, conocer y enseñar [2]. Por su parte Miller y Morris [4] plantean que el conocimiento surge de la integración de la teoría, la información y la experiencia.

Aunque, para Nonaka y Takeuchi [3] el conocimiento no puede enmarcarse en una sola definición, ya que aspectos como la cultura hacen que varíe el marco dentro del cual se da esta definición, así, por 
ejemplo, en la cultura oriental se considera que el conocimiento es principalmente algo tácito, difícil de encontrar, difícil de ver, difícil de entender y aún más difícil de expresar; sin embargo, los occidentales tienen fuertes creencias de que el conocimiento es explícito, ya que por obligación, debe poder expresarse matemáticamente o de forma escrita, haciendo así que este sea fácil de transmitir.

Por lo tanto, cuando se inicia con la aplicación de la ingeniería del conocimiento, en cualquier ámbito, suele enmarcarse en una perspectiva, ya sea en la que enfatiza en los procesos individuales de cada trabajador y en sus conocimientos tácitos para traducirlos en explícitos, conocida como perspectiva oriental; o en la perspectiva que considera que las organizaciones están preocupadas por el conocimiento de las personas para capitalizar ese conocimiento y, tomar decisiones, llamada perspectiva norteamericana [5], pero a pesar de los avances logrados en la ingeniería del conocimiento se ha observado que su uso es restringido en los países en desarrollo debido a la deficiente infraestructura tecnológica, dificultades de comunicación y desconocimiento de avances en esta área de conocimiento [6].

Así que, el conocimiento comienza a categorizarse como conocimiento tácito y conocimiento explícito. A diferencia del conocimiento explícito, el conocimiento tácito es difícil de codificar y está vinculado a los individuos [7]. La estrategia de codificación significa que conocimiento tácito es transferido en forma explícita y codificada y luego almacenada en bases de datos, bibliotecas, y archivos en los servidores físicos o digitales [8]. A pesar de esto, el hecho de que exista la tecnología de la información adecuada, no garantiza que el conocimiento va a fluir libremente a través del medio.

En el caso de una organización, los aspectos culturales son fundamentales, ya que es usual que, en las diferentes áreas o unidades estratégicas de negocio, haya un egoísmo con el conocimiento, por lo que deben emprenderse acciones encaminadas a fomentar una cultura de unidad empresarial [2]. En una organización innovadora las personas trabajan juntas para crear algo nuevo; desde una perspectiva empresarial, la pregunta es: ¿cómo manejar ese conocimiento individual eficientemente con el fin de satisfacer las necesidades del cliente? [7].
Según Alavi y Leidner [8], la ingeniería del conocimiento es un proceso sistematizado que permite la adquisición, organización y comunicación del conocimiento tanto tácito como explícito de manera efectiva. Además, se argumenta que este término está usualmente asociado con las tecnologías de la información y el conocimiento, ya que este es el recurso que más facilita el acceso a conocimiento generado por otros individuos o grupos, y permite compartirlo de manera fácil y ágil [2].

La ingeniería del conocimiento se ha convertido en una estrategia empresarial que se relaciona directamente con el grado de participación en el mercado. Pero, para lograr un alto grado de participación, es de gran utilidad emplear una valiosa herramienta de la que puede disponer cualquier empresa sin importar su objeto social, que es la investigación de mercados [5].

\section{¿Cuál es el papel de la ingeniería del conocimiento en la investigación de mercados?}

La investigación de mercados tiene un papel fundamental en la actualidad, ya que contribuye con la globalización y con la innovación, por lo que es clave entender que, para realizar una investigación acertada se requiere implementar la ingeniería del conocimiento y así orientar la investigación por el camino correcto, pero a su vez, una buena investigación, genera conocimiento que se puede transformar en innovación [9], por lo que se hace prioritario implementar más herramientas multidisciplinares en los procesos de investigación de mercados [10]. Dicha incorporación permite una dinámica empresarial más acorde con la realidad social y conduce a una planificación estratégica, mejor toma de decisiones de mercado y a su vez una mayor rentabilidad [11].

Sin embargo, la ingeniería del conocimiento puede llegar a ser compleja durante la estructuración de una investigación de mercados, ya que debe procurarse que esta investigación identifique e indague por separado las dos naturalezas principales del conocimiento: el conocimiento explícito y el conocimiento tácito, donde, siendo más específicos, el explícito es aquel que puede ser codificado, es más formal y usualmente está sistematizado, mientras que el conocimiento tácito es muy personal, y suele ser difícil para la persona que lo posee, transmitirlo con claridad [2]. Además, se plantea que una persona 
tiene más conocimiento del que puede transmitir, por lo que, este es el conocimiento sobre el que se dificulta más indagar y obtener información valiosa [2].

Frente a los retos de la investigación de mercados en este campo, se observa la necesidad de procesar grandes cantidades de información con el fin de extraer el conocimiento necesario para mejorar el desarrollo del proyecto y potencializar una mejor relación con los clientes, este reto se da ya que el análisis tradicional de esta información se ha mostrado ineficaz en costo y tiempo [12-13], por lo que se requieren nuevas alternativas que permitan construir perfiles de comportamiento de los consumidores, mostrando la dinámica de sus necesidades y motivaciones [14].

Actualmente, la economía ha cambiado, entrando en un período donde las empresas que más impactan el mercado son las que hicieron un gran cambio, desde la organización basada en la autoridad y el control, estructurada en departamentos y divisiones, hasta la organización basada en el conocimiento, es decir, la organización de los especialistas del conocimiento [15]. Por esto, en especial en los países desarrollados, las investigaciones de mercados juegan un papel fundamental en las empresas, ya que ayudan en la generación de estrategias con base en información de primera mano. Pero existen algunas compañías que aún no contemplan la inversión en investigación como un aspecto fundamental, ya que no comprenden que el uso y la generación de conocimiento aplicado a las organizaciones, genera resultados en el largo plazo [9].

Por su parte, Knight y Dalgic [16] complementan este planteamiento, argumentando que las capacidades de mercadeo que posee una empresa, incluyendo la importante investigación de mercados, tiene inherente a su desarrollo, el conocimiento, comenzando por el conocimiento del poder de la marca y lo que esta refleja en el mercado, además es obligación para una empresa generar y actualizar constantemente el conocimiento que posee acerca de sus productos, sus precios, su plaza y su promoción, para poder llegar a ser exitosa.

Una empresa de éxito hace bien en mantener y gestionar a sus clientes proporcionando una serie de atractivos servicios personalizados que satisfagan las necesidades de los mismos. Esto es debido a la premisa que plantea que es menos costoso mantener a los clientes actuales brindándoles un mejor producto y un mejor servicio, que hacer esfuerzos para atraer nuevos clientes [17]. Por lo tanto, una empresa debe comprender las necesidades de sus clientes existentes, satisfacer sus necesidades y construir relaciones sólidas basadas en una buena atención, para así llegar a forjar relaciones más estrechas y profundas y maximizar el valor y el tiempo que permanece un cliente en una organización, siendo la investigación de mercados una de las mejores herramientas para lograrlo [18].

En el caso en que la investigación de mercados la realiza directamente la empresa, esta debe ir ligada al concepto de conocimiento organizacional, el que consiste en una mezcla dinámica de las experiencias, los valores, la información y las ideas de individuos, grupos de trabajo y expertos. Dicho contexto se hace relevante ya que, a partir de esta información, se estructura de forma más robusta el proceso de investigación de mercados, en los casos en que tiene en cuenta la estructura interna de la organizacion. También es valioso a nivel externo, debido a que las acciones que la empresa realiza, afectan su entorno, y dichas acciones son el reflejo del conocimiento organizacional [2].

Para una empresa el hecho de aplicar la ingeniería del conocimiento, principalmente en los aspectos comerciales y de mercadeo, es considerado una de las capacidades más importantes que la empresa pueda desarrollar en su búsqueda por generar ventaja competitiva a través de las tecnologías de información [19]. Esto debido a que es necesario aprender a gestionar efectivamente el conocimiento que se tiene sobre los clientes y el conocimiento que los pueda beneficiar, lo que se denomina como capital relacional, además es fundamental saber explotarlo, difundirlo y aplicarlo [20]. Pero lo más importante es poder generar nuevo conocimiento y permitir que este fluya libremente por medio de la organización, lo cual puede lograrse mediante el desarrollo de las relaciones corporativas con otras entidades de manera estratégica y mediante el fortalecimiento de las redes de trabajo [21].

Según Rodríguez [22] toda empresa deberá entonces, diseñar un modelo para realizar efectivamente la ingeniería del conocimiento, y estos modelos 
suelen ser: a) modelos para almacenamiento, acceso y transferencia de conocimiento, que se enfoca en la creación de metodologías para almacenar el conocimiento que posee la organización; b) modelos con énfasis en el desarrollo de una cultura organizacional adecuada para la ingeniería del conocimiento; y c) modelos con énfasis en la tecnología, en los que se destacan el desarrollo y la utilización de sistemas y herramientas tecnológicas para la gestión del conocimiento.

Briceño \& Bernal [5] proponen otros modelos, que se agrupan según las perspectivas: a) modelos de enfoque técnico: sistematización de los conocimientos caracterizados por enfatizar en aspectos prácticos; $b$ ) modelos de enfoque economicista: enfatizan en que las personas son individuos que deben maximizar su bienestar a partir de unos recursos escasos y el conocimiento es uno de estos recursos; c) modelos de enfoque filosófico; d) modelos de enfoque social: enfatizan la importancia de los aspectos sociales para generar y compartir conocimiento; y e) modelos de enfoque sintético: muestra la existencia de varios puntos de vista como una forma de abordar la ingeniería del conocimiento.

Carson y Gilmore [23] hacen énfasis en que el foco de la ingeniería del conocimiento, en el proceso de la generación de nuevo conocimiento, es el cooperativismo; visto como las relaciones al interior de la empresa, las relaciones y cooperaciones con los grupos de interés, la cooperación con otras empresas la combinación entre recursos y procesos tangibles e intangibles y el reto que implica para toda empresa comunicar lo que desea proyectar.

Para el caso corporativo, Davenport \& Prusak [24] plantean que todas las organizaciones deben comenzar a implementar la ingeniería del conocimiento, ya que las empresas todo el tiempo están generando y usando el conocimiento. A medida que las organizaciones interactúan con sus entornos, absorben información, la convierten en conocimiento y desarrollan estrategias acordes a sus experiencias, valores y normas internas, por esto se dice que las empresas exitosas sienten y responden. Sin conocimiento, una organización no se podría organizarse a sí misma ni responder a los cambios de su entorno.

Por esto se dice que no importa si la empresa tiene la tecnología de punta para realizar sus procesos, o si tienen un sistema de reingeniería o incluso si realizan investigaciones de mercado frecuentemente. Si una empresa no tiene como base la ingeniería del conocimiento, no puede generar innovaciones que le permitan continuar en un mercado cada vez más competitivo [9]. Pero para desarrollar ingeniería de conocimiento que facilite la innovación y evite la fuga de conocimiento, hay que estructurar, ejecutar y controlar adecuadamente los métodos de gestión internos y las redes de trabajo en las que la empresa participa, convirtiendo estos en un factor de estabilidad y un aporte fundamental para generar ventaja competitiva [25].

Así que, cuando la ingeniería del conocimiento es aplicada correctamente, se logra que el conocimiento se vuelva de común entendimiento en todos los niveles de la empresa, y es importante destacar que la gran mayoría del conocimiento generado en una organización, es de naturaleza tácita y es exclusivo de la compañía por lo que es muy difícil que sea usado por otras empresas, por ende, la ingeniería del conocimiento se convierte en una ventaja competitiva [26].

Los componentes principales de la ingeniería del conocimiento incluyen bases de datos relacionales, sistemas de almacenamiento de datos, soporte de decisiones y sistemas de análisis de datos multidimensionales [27]. Otros estudios han incorporado técnicas de ingeniería de valor (Value Engineering Technique) para reducir los costos y mejorar la precisión de las investigaciones de mercado [28] y redes neuronales artificiales utilizadas para la clasificación y evaluación en la similitud de preferencias entre consumidores de varios países [29]. Otro ejemplo de esta aplicación son sistemas de ingeniería de conocimiento diseñados para la segmentación de mercados agrícolas [30] y en términos genéricos sistemas que buscan generar recomendaciones a las compañías en sus procesos de comercialización y ventas a partir de la búsqueda frecuente y rápida de los indicadores del mercado, planteando como reto la mejora en las tareas de adquisición del conocimiento de estas herramientas tecnológicas [31].

Además, se requieren sistemas que mejoren los procesos de diseño de productos a partir de la identificación de las necesidades individuales de los clientes de una forma rápida y a menores costos [32]. 
En el entorno actual, las empresas sirven a los clientes o grupos de clientes mediante la utilización de una variedad de canales comercialmente, por lo que, para entender a sus clientes con una visión unificada, las empresas intentan integrar una gran cantidad de datos recogidos mediante múltiples canales [33], incluyendo la navegación web, las quejas, sugerencias, reclamos y las características demográficas, entre otros. Esto suele complicarse debido a que las empresas dividen a sus clientes en numerosos grupos con preferencias similares y examinan distintas características de cada grupo para determinar los segmentos más rentables [34], en dicho proceso se ha optado por robustecer los procesos de validación de la información recolectado y por la triangulación metodológica a partir de la combinación de diversas diseños cuantitativos y cualitativos [35], entre ellas las técnicas proyectivas como alternativas válidas, fiables y significativas para abordar problemas de investigación de mercados [36].

Para aplicar la ingeniería del conocimiento específicamente en el entendimiento de los clientes, el proceso más ampliamente aceptado y aplicable es iniciar haciendo una segmentación de estos, así: (1) Elegir una base para la segmentación y seleccionar las variables apropiadas, por ejemplo, las demográficas o de comportamiento; (2) Utilizar el análisis multivariado a clientes juntos o divididos en grupos; (3) Evaluar y validar los resultados; y (4) Analizar los resultados en términos económicos [34].

Por otro lado, autores como Shaw, Subramaniam, Tan y Welge [37] argumentan que la segmentación de clientes en la actualidad no es útil, ya que las decisiones como promociones, canales de distribución de marketing y la publicidad en los medios de comunicación, basados en el tradicional enfoque de segmentación dan como resultado una tasa de respuesta pobre y un aumento de los costos. Los clientes de hoy tienen tan variados gustos y preferencias que no es posible agruparlos en grandes poblaciones homogéneas para desarrollar estrategias de marketing, de hecho, cada cliente quiere ser servido según sus necesidades individuales y únicas. En los últimos años, el advenimiento de la tecnología de la información ha transformado la forma de hacer marketing y el cómo las empresas gestionan la información sobre sus clientes. La disponibilidad de grandes volúmenes de datos sobre clientes, ha creado tanto oportunidades para las empresas como retos, ya que el aprovechamiento de los datos y la obtención de ventajas competitivas se ha vuelto complejo [37].

Por ejemplo, la internet y la World Wide Web han hecho que el proceso de recolección de datos sea mucho más fácil, aumentando el volumen de información disponible para las empresas. Por un lado, muchas organizaciones han comprendido que la información disponible en estas enormes bases de datos es clave para apoyar las diversas decisiones organizativas, en particular, el conocimiento sobre los clientes, ya que es un factor crítico para la función de marketing, pero, gran parte de este conocimiento útil está oculto y sin explotar. Por otro lado, la intensa competencia y las mayores opciones disponibles para los clientes han creado nuevas presiones en los tomadores de decisiones de marketing y ha surgido la necesidad de administrar a los clientes en una relación a largo plazo. Este nuevo fenómeno, denominado gestión de las relaciones con los clientes, requiere que las organizaciones basadas en las preferencias del cliente, adapten sus productos y servicios e interactúen con sus clientes [18].

Otro reto importante es la minería web, con internet emergiendo como el nuevo canal de distribución de mercancías, de promoción de productos, de manejo de transacciones y de coordinación de procesos de negocio. Sin embargo, los múltiples formatos de los datos y la naturaleza distribuida del conocimiento en la web hacen un reto para recoger, descubrir, organizar y administrar el conocimiento de una manera que es útil para la toma de decisiones de marketing. Como la comercialización depende cada vez más de la web para los datos del cliente, la necesidad de la minería web puede abordarse como un problema importante de gestión e ingeniería de conocimiento y comercialización [37].

Mientras las organizaciones se mueven hacia la gestión de relaciones con clientes, la función de marketing, como la primera línea para interactuar con los clientes, es la responsable de afrontar estos cambios. Hay un creciente reconocimiento que pueda hacer efectiva la gestión de la relación basada solamente en un verdadero entendimiento de las necesidades y preferencias de los clientes. En estas condiciones, las herramientas de minería de datos pueden ayudar a descubrir el conocimiento oculto y 
entender mejor al cliente, mientras que un esfuerzo en la gestión del conocimiento sistemático puede canalizar el conocimiento en estrategias de marketing efectivas. Esto hace que el estudio de la extracción y la gestión de conocimiento, sea especialmente valioso para la comercialización [37-38].

Desde 1992 se había planteado esta necesidad, al respecto Holtz [39] mencionaba que las base de datos de marketing, caracterizadas por estrategias de marketing que tienen como base la gran cantidad de información disponible en las bases de datos del cliente, se han vuelto cada vez más populares y la mayoría de las organizaciones han acumulado enormes bases de datos sobre sus clientes y sus transacciones de compra, pero, debido a la falta de adecuadas herramientas y técnicas para analizar estas enormes bases de datos, una gran cantidad de información del cliente y sus patrones de compra es permanentemente ocultada.

El marketing basado en el conocimiento, que utiliza la ingeniería del conocimiento con las herramientas adecuadas de minería de datos, aborda esta necesidad y ayuda a aprovechar conocimientos ocultos en bases de datos. Hay tres áreas principales de la aplicación de minería de datos para el marketing basado en el conocimiento: 1) Perfiles de los clientes, 2) análisis de las desviaciones y 3 ) análisis de tendencias [37].

La selección de algoritmos de minería de datos, la formación de hipótesis, la evaluación del modelo y el refinamiento, son componentes clave de este proceso de descubrimiento, porque se necesitan ciclos de pruebas y errores para producir progresivamente los conocimientos más útiles de la minería de datos [40] y también puede ser útil para asegurar que el proceso puede finalmente generar conocimiento de valor para la organización. Uno de los retos de la investigación es hacer este proceso más estructurado y así mejorar la productividad de los esfuerzos de minería de datos.

Un segundo reto es gestionar el conocimiento producto de este que cruza las fronteras organizacionales y que se distribuye por medio de socios de la cadena de suministro, por lo que gestionar este conocimiento requiere de una gran organización y de los esfuerzos intersectoriales a nivel industrial. Los temas clave para generar la investigación son el desarrollo de modelos de gestión e ingeniería de conocimiento entre organizaciones apropiadas, la protección de los derechos del conocimiento y la distribución de los beneficios del conocimiento entre los socios [37], [41].

Un tercer reto para la investigación de la gestión e ingeniería del conocimiento son las múltiples clasificaciones que pueden realizarse, tanto con clientes como con procesos, ocasionando así, que por ejemplo los clientes puedan pertenecer a más de una categoría. Las técnicas de minería de datos actuales han demostrado estar limitadas en el manejo de membresías a múltiples clases [42]. La creciente complejidad de las preferencias del cliente hace que este tema sea particularmente relevante para los vendedores, pues pueden encontrar a clientes que pertenecen a varias clasificaciones, por lo que necesitan herramientas de segmentación confiables para obtener un mayor conocimiento sobre los clientes, en lugar de simplificar las clases y perder información valiosa [43].

Los campos de la gestión e ingeniería del conocimiento y la minería de datos siguen evolucionando y así presentan desafíos interesantes para los investigadores y profesionales, con implicaciones para la función de marketing. Aun cuando se presenta un marco integrado para la gestión e ingeniería del conocimiento en el contexto del marketing, es evidente la existencia de retos críticos de investigación a abordar [37], [44].

\section{APLICACIÓN DE LA INGENIERÍA DEL CONOCIMIENTO EN EL CASO MOBILE LEARNING}

Los factores que explican el proceso mediante el cual una tecnología cualquiera es adoptada o no por un usuario en particular se recogen en diferentes modelos relativos a la adopción tecnológica. Los modelos de adopción tecnológica intentan predecir el uso futuro de una tecnología nueva, o explicar el uso actual de una tecnología existente por parte de un individuo [45].

En 1989, Davis introdujo por vez primera el Modelo de Adopción Tecnológica (TAM, por sus siglas en inglés) para explicar la aceptación y el uso de tecnologías de la información. El TAM se basa en la Teoría de la Acción Razonada, la que explica el comportamiento de un individuo a partir de su 
intención de desempeñar el comportamiento; según esta teoría, las creencias influyen en las actitudes, las cuales conducen a las intenciones, generando la conducta [46]. El TAM adoptó esta lógica de razonamiento, y la particularizó para la conducta específica adopción de tecnologías [47].

Según el TAM, la facilidad de uso percibida y la utilidad percibida son los dos predictores de la variable independiente intención de uso, la que desde la TRA se supone estrechamente vinculada con el comportamiento real [48]. La facilidad de uso percibida se refiere al grado en que un individuo cree que el uso de un sistema particular requiere poco esfuerzo, y la utilidad percibida hace referencia al grado en que un individuo cree que el uso de una determinada tecnología mejorará el desempeño de sus actividades [49]. Adicionalmente, el TAM establece una relación entre la facilidad de uso percibida y la utilidad percibida. En este sentido, la facilidad de uso percibida y la utilidad percibida tendrán un efecto directo sobre la intención de uso, o indirecto sobre la misma afectando a la otra variable en mención [50].

Para el proyecto realizado en el ITM denominado aceptación del mobile learning en los estudiantes universitarios, se desarrolló una investigación aplicada de carácter transversal por medio de una triangulación metodológica que incorpora diseños metodológicos cuantitativos representados en cuestionarios autoadministrados y cualitativos mediante grupos focales y entrevistas en profundidad, aplicando los principios de la ingeniería del conocimiento. Se identificaron las diferentes herramientas de adquisición de conocimiento que podrían aplicarse a los diferentes públicos objetivos seleccionados, con esto, se pretendía obtener su nivel de percepción sobre el uso de tecnologías móviles en entornos educativos.

El objetivo general del proyecto plantea "analizar los factores y variables críticas en los procesos de aceptación y uso del mobile learning por parte de los estudiantes del Instituto Tecnológico Metropolitano" por lo que el estudio se focaliza solo en los estudiantes del ITM debido a que este tipo de investigaciones de aceptación tecnológica deben llevarse a cabo en contextos socioculturales y educativos específicos. Este estudio no contemplaba la caracterización de los estudiantes de las instituciones de educación superior en general ya que está fuera del alcance de los objetivos y el tiempo planteado.

Adicionalmente, esta propuesta de investigación contempla un muestreo no probabilístico, escogiendo como método el muestreo por criterio y como población objetivo estudiantes del ITM. Además, esta propuesta investigativa plantea una triangulación metodológica (encuestas, grupos focales, entrevistas) ya que la implementación de este procedimiento de control permite una mayor garantía de la confiabilidad en los resultados al combinar varias metodologías de investigación en el estudio de un mismo fenómeno, fortaleciendo la construcción, consistencia e interpretación de resultados [51].

Estos mecanismos de adquisición de conocimientos se seleccionaron además teniendo en cuenta la etapa del proyecto en la cual era necesaria contar con los instrumentos de medición y al tipo de público a los que se iba a aplicar. Para esto se aplicaron las taxonomías de adquisición de conocimiento propuestas por Rodríguez-Lora [22], adicionalmente, estas técnicas deberían tener como propósito el de cumplir con el proceso de confiabilidad en los resultados de la investigación y de adquirir el conocimiento tanto tácito como explícito de los grupos de interés. Es por ello que se aplicaron, por medio de la triangulación los tres diseños metodológicos que se describen en la figura a continuación.

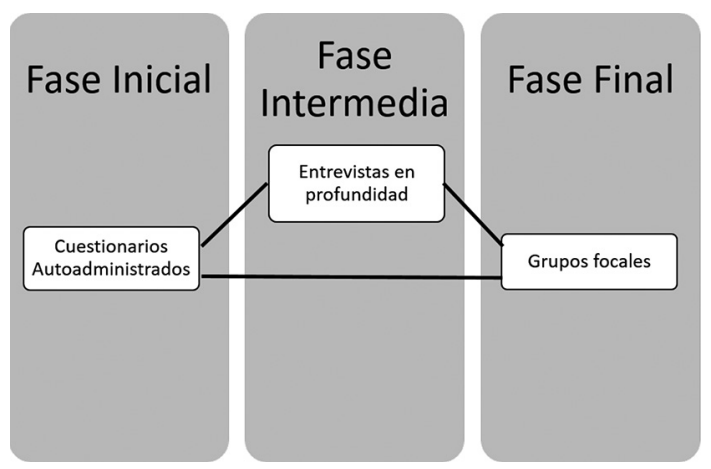

Figura 1. Triangulación de técnicas de adquisición de conocimiento.

\section{Cuestionarios autoadministrados}

Con el objetivo de recoger información de carácter cuantitativo, se aplicó un cuestionario tipo encuesta; 
buscando obtener de forma organizada los indicadores de las variables implicadas en el objetivo de la encuesta [52]. Este cuestionario se aplicó de forma autoadministrado buscando bajo coste, conveniencia, rapidez y facilidad de creación [53] y se realizaron a 657 estudiantes del ITM seleccionados por medio de un muestreo no probabilístico por criterio. Finalmente, la información recogida fue tabulada para facilitar el análisis de la información. Desde el punto de vista de la adquisición del conocimiento, el uso de esta herramienta permitió adquirir conocimiento del tipo explícito en una etapa inicial del desarrollo del proyecto.

\section{Grupos focales}

Los grupos focales son una técnica de adquisición de conocimientos mediante una entrevista grupal semiestructurada, con un objetivo particular; la que gira alrededor de una temática propuesta por el investigador [54]. Por medio de un espacio de opinión se pretende captar el sentir, pensar y vivir de los individuos, provocando auto explicaciones para obtener información cualitativa [55] y conocimiento tácito.

Los grupos focales se conformaron por grupos de 8 a 12 estudiantes activos del ITM, la duración del grupo focal fue alrededor de 90 minutos. Para la dinámica del grupo se procedió a socializar los objetivos del grupo focal, luego se contextualizó sobre el mobile learning y a partir de preguntas semiestructuradas se buscó examinar las experiencias, percepciones y opiniones de los estudiantes participantes frente a los diversos constructos que componen el Modelo de Aceptación Tecnológica.

\section{Entrevistas en profundidad}

La entrevista en profundidad está definida como el encuentro cara a cara entre el investigador y los entrevistados, buscando comprender las perspectivas que tienen estos últimos respecto de sus vidas, experiencias o situaciones [56], de un dominio de conocimiento en particular, en este caso, el dominio corresponde a las tecnologías móviles. En esta técnica, se explora, detalla y rastrea por medio de preguntas, cuál es el conocimiento que el experto posee y que es más relevante para los intereses de la investigación [57].

Si bien el estudio está dirigido a estudiantes del ITM, los docentes del ITM tienen un fuerte vínculo con sus procesos de aprendizaje por lo tanto aportan de forma valiosa a la investigación con sus apreciaciones y percepciones frente a la aceptación del mobile learning en los estudiantes, lo cual nos permite, por medio de las entrevistas en profundidad, examinar el impacto que podría traer esta herramienta a los procesos de enseñanza-aprendizaje que se llevan a cabo con los estudiantes. Lo anterior, muestra la necesidad de incorporar en el trabajo de campo de carácter cualitativo la participación de los docentes en las entrevistas en profundidad, orientándonos especialmente a docentes que incorporen prácticas en el aula relacionadas con tecnologías móviles como herramienta de interacción y objeto de enseñanzaaprendizaje, es por esto que en este caso se toma a los docentes como los expertos en el dominio. Asimismo, los jefes de programa y el personal administrativo de la plataforma virtual del ITM pueden brindar valiosa información para apoyar los resultados que se obtendrán con la aplicación del cuestionario autoadministrado a los estudiantes.

Para la investigación se realizaron quince entrevistas en profundidad, dirigidas a personal docente y administrativo del Instituto Tecnológico Metropolitano. Con el conocimiento obtenido a través de la aplicación de las diferentes herramientas se pretende identificar cual es el nivel de aceptación de los estudiantes del ITM frente al uso de los dispositivos móviles en los ambientes de enseñanza y aprendizaje. Adicionalmente, una vez obtenidos los resultados de los estudios, se podrá llevar este conocimiento a un esquema de representación que permita ver gráficamente como los factores y variables de estudio determinan dentro del proceso de aceptación o uso del mobile learning por parte de los estudiantes.

\section{DISCUSIÓN}

Es más común encontrar la fusión entre la aplicación de la gestión del conocimiento y la investigación de mercados en el contexto organizacional [58] como mecanismo para fortalecer los procesos de transferencia tecnológica entre las universidades y el sector empresarial [59], es el caso de los modelos para medir la satisfacción del cliente como mecanismo de seguimiento y plantear estrategias de mejora en los servicios ofrecidos [60] y de sistemas multiagente para mejorar el proceso de aprendizaje del empresario sobre las estrategias de investigación de mercados [61]; sin embargo, dicha fusión ha empezado a 
cobrar importancia en el campo educativo debido a la necesidad de mejorar el impacto del uso de nuevas herramientas de gestión tecnológica en las diferentes áreas de conocimiento [62], como es el caso de los objetos virtuales de aprendizaje como mecanismos para generar mayores posibilidades de interactividad y autonomía en el aprendizaje [63]. Además, la gestión del conocimiento puede convertirse en una herramienta para que desde el uso de TIC se generen mecanismos de inclusión social y educativa que puedan ser replicables en otros contextos educativos [64].

En el caso de estudio seleccionado, se planteó la necesidad de realizar triangulación de técnicas de adquisición de conocimiento, por lo que se vuelve prioritaria la aplicación de taxonomías de técnicas y herramientas para la ingeniería del conocimiento en la selección y aplicación de dichas técnicas [65], es por esto que se toma como fase inicial la recolección de cuestionarios autoadministrados, frente a los cuales se plantean diversas posibilidades de análisis, desde una perspectiva descriptiva se pueden tener información sobre las percepciones de los estudiantes, sus intereses, expectativas y usos posibles que puedan dar a las TIC [66], lo que se convierte en un insumo fundamental para iniciar cualquier estrategia educativa que involucre el uso de TIC e implique herramientas de gestión tecnológica [67], [68]. Adicionalmente, los diseños cuantitativos pueden ser usados para la recolección de datos que sirvan como insumo para la generación de modelos más estructurados en términos cuantitativos desde una perspectiva estadística, lo cual puede ir desde el cálculo de niveles de asociación entre los constructos del modelo de aceptación tecnológica [69], [70] hasta el planteamiento de modelos de ecuaciones estructurales alrededor de las variables que influyen en la adopción del mobile learning [71]. Asimismo, en su fase intermedia y fase final se propone la utilización de diseños metodológicos cualitativos, quienes permiten una mayor profundidad frente a aspectos puntuales de la temática, brindando la posibilidad de generar contraste entre las vivencias, opiniones y perspectivas del público objetivo, este tipo de diseños es pertinente para temáticas de corte más social, como es el caso de temáticas como el impacto del de TIC en la convivencia familiar [72].

Lo anterior es coherente con los retos de las universidades, los que apuntan a la necesidad de capitalizar las experiencias investigativas, hacer una mejor gestión del conocimiento generados y beneficiarse del nexo enseñanza-investigación, proporcionando a sus estudiantes mayores experiencias en este ámbito por medio del uso efectivo de las TIC [73]. En este mismo contexto, se observa que las tendencias en esta área del conocimiento se enfocan en temáticas como el aprendizaje colaborativo, el aprendizaje en red y las comunidades virtuales de aprendizaje [74], las cuales representan un verdadero potencial para el estudio de la adopción del mobile learning desde una perspectiva de ingeniería del conocimiento.

\section{CONCLUSIONES}

La ingeniería del conocimiento dejó de pertenecer exclusivamente a la computación y son hoy diversas áreas que la están empleando como un mecanismo para recuperar conocimiento crítico, identificar conocimiento valioso y manipular conocimiento relevante en las organizaciones. Para ello, la investigación de mercados está empleando sus beneficios en aras de conocer aquello que es importante y relevante de sus clientes y realizar sus mayores esfuerzos en aquello que han identificado que es de valor para ellos y que está relacionado en la satisfacción de sus necesidades.

Desde la perspectiva del estudio realizado por el Instituto Tecnológico Metropolitano, conocer que piensan sus estudiantes, su nivel de aceptación hacia la incorporación de nuevas tecnologías debe tener un propósito misional que debe ir más allá que simplemente incorporar tecnología.

La documentación realizada frente a la recolección de información en el caso investigativo planteado permite que el diseño de los aplicativos móviles orientados a potencializar el uso del mobile learning partan de las verdaderas necesidades, experiencias y conocimientos de los usuarios potenciales de las herramientas, lo que genera una mayor garantía frente al posicionamiento y uso de los dispositivos móviles.

Entre los hallazgos de la investigación se logró identificar que los principales factores de influyen en la aceptación del mobile learning en los estudiantes universitarios del ITM son: autonomía en el aprendizaje, autoeficacia percibida, facilidad de 
uso percibida, preparación del instructor y utilidad percibida, lo que puede convertirse en un punto de partida que puede servir a otras instituciones de educación superior para que a partir de la metodología replicable que se plantea se puedan contrastar los resultados obtenidos y de esta forma se generen futuros estudios comparativos que permitan entender que otros factores socioculturales adicionales influyen en la aceptación del aprendizaje por medio de dispositivos móviles.

Entre los trabajos futuros que se desprenden de la investigación planteada se observa la necesidad de proponer nuevos modelos conceptuales que articulen y compilen los conocimientos generados a partir de los factores de aceptación que se identificaron con el fin de generar procesos de validación más robustos frente a este tipo de procesos de adopción del aprendizaje por medio de dispositivos móviles.

\section{RECOMENDACIONES}

Queda claro entonces que la ingeniería del conocimiento puede aplicarse a diferentes áreas del saber tales como, la ingeniería, la administración, la sociología, la psicología, la geología, entre otros. Su campo es lo suficientemente amplio para abarcar las diferencias y particularidades de cada una de estas, dependerá de la habilidad que desarrolle el ingeniero del conocimiento en identificar el tipo de problemática, de conocimiento, de técnicas y herramientas para la adquisición y representación lo que definirá el éxito de un proyecto y de los resultados que este tenga.

Sería importante entonces, que los investigadores universitarios, se entrenen en esta área como un mecanismo para la mejora de sus investigaciones y de los resultados que se obtengan. Asimismo, en la formación de los estudiantes, para que identifiquen los diferentes tipos de conocimiento y estén en capacidad de relacionar estos a las herramientas idóneas para adquirirlos y representarlos.

Es así que, desde el campo académico, la ingeniería del conocimiento podría cobrar un interés particular ontológica y epistemológicamente, al reconocer los diferentes tipos de conocimiento y tratarlos como debería hacerse: como una representación del conocer y del saber de los individuos, que se complementan entre sí, pero que sus desarrollos, objetos y accionar son completamente diferentes.

\section{REFERENCIAS}

[1] R.H. Assudani. "Catching the chameleon: understanding the elusive term "knowledge". Journal of Knowledge Management. Vol. 9 $\mathrm{N}^{\circ}$ 2, pp. 31-44. 2015.

[2] C.T. Small and A.P. Sage. "Knowledge management and knowledge sharing: A review. Knowledge Management". Information Knowledge systems management. Vol. $5 \mathrm{~N}^{\circ}$ 3, pp. 153-169. 2006.

[3] I. Nonaka and H. Takeuchi, "The knowledgecreating company. How Japanese companies create the dynamics of innovation". Oxford University Press. New York, Estados Unidos. 1995.

[4] W.L. Miller and L. Morris. "Fourth generation R\&D: Managing knowledge, technology, and innovation". Wiley India Pvt. Limited. Delhi, India. 2008

[5] M.A. Briceño y C.A. Bernal. "Estudios de caso sobre la gestión del conocimiento en cuatro organizaciones colombianas líderes en penetración de mercado". Estudios Gerenciales. Vol. 6 N$^{\circ}$ 26, pp. 173-193. 2010.

[6] S. Schlobach, V. De Boer, C. Guéret; S. Boyera and P. Cudré-Mauroux. "From Knowledge Engineering for Development to Development Informatics”. En: Knowledge Engineering and Knowledge Management, pp. 18-29. Springer International Publishing. 2014.

[7] T. Lehtimäki, H. Simula and J. Salo. "Applying knowledge management to project marketing in a demanding technology transfer project: Convincing the industrial customer over the knowledge gap". Industrial Marketing Management. Vol. 38 N$^{\circ}$ 2, pp. 228-236. 2009.

[8] M. Alavi and D. Leider. "Knowledge management systems: Emerging views and practices from the field In Systems Sciences". Proceedings of the 32nd Annual Hawaii International Conference. Hawaii, Estados Unidos. 1999.

[9] P. Nath and N. Mrinalini. "Knowledge Management in Research and Technology Organizations in a Globalized Era". Perspectives on Global Development and Technology. Vol. $7 \mathrm{~N}^{\mathrm{o}}$ 1, pp. 37-54. 2008.

[10] S.A. Matei, B. Britt, E. Bertino and J. Foote. "The Trajectory of Current and Future 
Knowledge Market Research: Insights from the First KredibleNet Workshop". En: Roles, Trust, and Reputation in Social Media Knowledge Markets Springer International Publishing, pp. 169-196. 2015.

[11] M. Regberg. "Understanding the value of market research". ISA TECH/EXPO Technology Update Conference Proceedings. Vol. 401, pp. 79-84. 2000.

[12] S. Desa and T.A. Munger. "RepresentationBased Methodology for Developing High-Value Knowledge Engineering Software Products: Theory, Application, and Implementation". Journal of Computing and Information Science in Engineering. Vol. $13 \mathrm{~N}^{\mathrm{o}}$ 4, pp. 1-19. 2013.

[13] A. Gama y A.A. Gaitán. "Intellectual Assets and Knowledge Engineering Method: A Contribution". 7th International Conference on Knowledge Management in Organizations: Service and Cloud Computing, pp. 209-218. Springer Berlin Heidelberg. 2013.

[14] P. Kutty. "Blending market research and user research activities". Interactions. Vol. 22 $\mathrm{N}^{\circ}$ 3, pp. 68-70. 2015.

[15] D. Rodríguez. "Modelos para la creación y gestión del conocimiento: Una aproximación teórica”. Educar. Vol. 37, pp. 25-39. 2006.

[16] G.A. Knight y T. Dalgic. "Market orientation, marketing competence, and the international performance of the firm". Summer American Marketing Association Conference. Chicago, Estados Unidos. 2000.

[17] J.Peppard. "Customer relationship management CRM. Financial services". European Management Journal. Vol. $18 \mathrm{~N}^{\circ} 3$, pp. 312327. 1999.

[18] D. Peppers; M. Rogers y B. Dorf. "Is your company ready for one-to-one marketing". Harvard Business Review. Vol. $77 \mathrm{~N}^{\circ} 1$, pp. 151-160. 1999.

[19] V.,Hrgovcic; W. Utz y R. Woitsch. "Knowledge engineering in future internet". Springer Berlin Heidelberg, pp. 100-109. 2009.

[20] D.B. Arnett y V. Badrinarayanan. 2005. "Enhancing Customer-Needs-Driven CRM Strategies: Core Selling Teams, Knowledge Management Competence, and Relationship Marketing Competence". Journal of Personal Selling \& Sales Management. Vol. $25 \mathrm{~N}^{\circ} 4$, pp. 329-343. 2005
[21] R.K. Srivastava; T.A. Shervani y L. Fahey. "Marketing, business processes, and shareholder value: An organizationally embedded view of marketing activities and the discipline of marketing". The Journal of Marketing, Vol. 63 No 4, pp. 168-179. 1999.

[22] V. Rodríguez-Lora. "Diseño e implementación de un modelo de conocimientos sobre las cuencas petrolíferas de Colombia”. Medellín: Trabajo de grado presentado para obtener el título de Magíster en Ingeniería, Universidad EAFIT. Medellin, Colombia. 2012.

[23] A. Gilmore y D. Carson. "Quality improvements in a services marketing context". Journal of Services Marketing. Vol. 7. № 3, pp. 59-71. 1993.

[24] T.H. Davenport y L. Prusak. "Working knowledge: How organizations manage what they know". Boston: Harvard Business Press. 1998

[25] J.J.Pinto. "Sistemas de gestión de competencias basados en capacidades y recursos y su relación con el sistema seci de gestión del conocimiento, realizadas por las pequeñas empresas del urola medio España”. Estudios Gerenciales, Vol. 23 No 105, pp. 13-38. 2007.

[26] Q. Liu y T. Wang "The formative mechanism of marketing capability. The View Of Organizational Learning and Knowledge Management". 2nd International Symposium On Information Science and Engineering, ISISE. Shangai, China. 2009.

[27] W. Kim; K. Chae; D.S. Cho; B. Choi; M. Kim, K. Lee y H. Yong. "Component-based knowledge engineering architecture". Journal of Object-Oriented Programming, Vol. 12 $\mathrm{N}^{\circ}$ 6, pp. 40-48. 1999.

[28] T. M. Cook. "Implementing Market Research into Customer Focused Value Engineering" No 960007. SAE Technical Paper. 1996.

[29] A. P. Azcarraga; M. H. Hsieh y R. Setiono. "Market research applications of artificial neural networks". Evolutionary Computation. IEEE World Congress on Computational Intelligence, IEEE. Hong Kong, China. 2008.

[30] J. D. Reece; J. Sumberg y L. Pommier. "Matching technologies with potential end users: a knowledge engineering approach for agricultural research management". Journal of Agricultural Economics. Vol. 55 $\mathrm{N}^{\mathrm{o}}$ 1, pp. 25-40. 2004. 
[31] A. Felfernig; M. Mandl y M. Pumschubert. Empirical knowledge engineering: cognitive aspects in the development of constraintbased recommenders. En: Trends in Applied Intelligent Systems. Springer Berlin Heidelberg, pp. 631-640. 2010.

[32] L. Tai; D. Li, T. Zhao y T. Jiang. "Product Rapid Response Design Based on Knowledge Engineering". In Advanced Materials Research. Vol. 271, pp. 563-568. 2011.

[33] T. Ruf y T. Kirsche. "Data Refinement in a Market Research Applications' Data Production Process". Data Management in a Connected World, pp. 159-163. 2005.

[34] S. Ha. "Applying knowledge engineering techniques to customer analysis in the service industry". Advanced Engineering Informatics. Vol. $21 \mathrm{~N}^{\circ}$ 3, pp. 293-301. 2007.

[35] A. Takhar-Lail y A. Ghorbani. "Market Research Methodologies: Multi-Method and Qualitative". IGI Global, pp. 300. 2015. ISBN: 9781466663718.

[36] E. Ramsey. "Application of projective techniques in an e-business research context: a response to 'Projective techniques in market research-valueless subjectivity or insightful reality?". International Journal of Market Research. Vol. $47 \mathrm{~N}^{\circ}$ 3, pp. 551-573. 2006.

[37] M.J. Shaw; C. Subramaniam; G.W. Tan y M.E. Welge. "Knowledge management and data mining for marketing". Decision Support Systems. Vol. 31 N $^{\mathrm{o}}$ 1, pp. 121-137. 2001.

[38] K. Fernández-Aguirre; M.I. Landaluce; A. Martín y J.I. Modroño. "Data Mining of an On-line Survey-A Market Research Application". En: Data Analysis, Machine Learning and Applications. Springer Berlin Heidelberg, pp. 183-191. 2008.

[39] H. Holtz. "Databased Marketing-Every Manager's Guide to the Super Marketing Tool of the 21st Century". London: John Wiley \& Son, INC. 1992.

[40] F.R. Lin y M.J. Shaw "Active training of backpropagation neural networks using the learning by experimentation methodology". Annals of Operations Research. Vol. 75, pp. 105-122. 1997.

[41] L. Diez, A. Valencia y E. Villa. "Promoción de la cultura investigativa como motor de desarrollo económico y social: una visión sistémica". Revista Espacios. Vol. 36, № 1, 2015.
[42] W.E. Spangler; J.H. May y L.G. Vargas. "Choosing data-mining methods for multiple classification: representational and performance measurement implications for decision support". Journal of Management Information Systems. Vol. 16 No 1, pp. 37-62. 1999.

[43] S.S. Kraljevic. "The usage of market research during the segmentation of buyers". Informatologia. Vol. $39 \mathrm{~N}^{\circ} 3$, pp. 170-176. 2006.

[44] S. M. Bjerrisgaard y D. Kjeldgaard. "The way you see is what you get. Market research as modes of knowledge production". En: Marketing Management. Routledge, pp. 231245. 2012.

[45] V. Venkatesh y H. Bala. Technology acceptance model 3 and a research agenda on interventions. Decision Sciences. Vol. 39 $\mathrm{N}^{\mathrm{o}}$ 2, pp. 273-315. 2008.

[46] M. Fishbein, y I. Ajzen. "Belief, attitude, intention, and behavior: An introduction to theory and search". Addison-Wesley Pub (Sd), pp. 480. 1975. ISBN: 978-0201020892.

[47] M.Abbad; D. Morris y C. De Nahlik. "Looking under the Bonnet: Factors Affecting Student Adoption of E-Learning Systems in Jordan". International Review of Research in Open and Distance Learning. Vol. $10 \mathrm{~N}^{\circ}$ 2, 2009. ISSN 14923831. Fecha de consulta: 15 de septiembre de 2016. DOI: 10.19173/irrodl. v10i2.596.

[48] W.R. King y J. He. "A meta-analysis of the technology acceptance model". Information \& Management. Vol. $43 \mathrm{~N}^{\circ}$ 6, pp. 740-755. 2006.

[49] F.D. Davis. "Perceived usefulness, perceived ease of use, and user acceptance of information technology". MIS Quarterly, pp. 319-340. 1989.

[50] M.R. Martínez-Torres; S.L. Toral; F.B. García; S. Vázquez; M. Oliva y T. Torres. "A technological acceptance of e-learning tools used in practical and laboratory teaching, according to the European higher education area". Behaviour \& Information Technology. Vol. $27 \mathrm{~N}^{\mathrm{o}}$ 6, pp. 95-505. 2008.

[51] D.S. Donolo. "Triangulación: Procedimiento incorporado a nuevas metodologías de investigación”. Fecha de consulta: 24 de octubre de 2016. URL: http://www.eumed.net/ 
tesis-doctorales/2009/njlg/index.htmhttp:// www.revista.unam.mx/vol.10/num8/art53/ int53.htm

F.G. Alcaraz; A.A. Espín; A.H. Martínez y M.M. Alarcón. "Diseño de Cuestionarios para la recogida de información: metodología y limitaciones". Revista Clínica de Medicina de Familia. Vol. 5, pp. 232-236.

[53] A. Suárez; N. García y M.B. Álvarez. "La interactividad en cuestionarios autoadministrados. Influencia en la experiencia del encuestado". Cuadernos de Economía y Dirección de la Empresa. Vol. 12 № 41, pp. 69-92. 2009

[54] J. Escobar y F.I. Bonilla-Jiménez. "Grupos focales: una guía conceptual y metodológica". Cuadernos Hispanoamericanos de Psicología. Vol. $9 \mathrm{~N}^{\mathrm{o}}$ 1, pp. 51-68. 2009.

[55] A. Hamui y M. Varela. "La técnica de grupos focales". Inv Ed Med, Vol. 2 No 1, pp. 55-60. 2013.

[56] N.J. López. "Entrevista en profundidad y focalizada". Fecha de consulta: 15 de septiembre de 2016. URL: URL: http://www.eumed.net/ tesis-doctorales/2009/njlg/index.htm

[57] B. Robles. "La entrevista en profundidad: una técnica útil dentro del campo antropofísico". Cuicuilco. Vol. 18 N $^{\circ}$ 52, pp. 39-59. 2011.

[58] J. Hoyos y A. Valencia. "El papel de las TIC en el entorno organizacional de las pymes". Trilogía. Ciencia, Tecnología y Sociedad. Vol. 7, pp. 105-122, 2012. URL: http:// itmojs.itm.edu.co/index.php/trilogia/article/ download/378/373

[59]. E. Villa; E. Picón; A. Valencia-Arias y C. Jiménez. "Analysis of University Management of Emerging Technologies and Recommendations for Developing Countries". Turkish Online Journal of Educational Technology, Vol. 16. $\mathrm{N}^{\mathrm{o}}$ 2, pp. 1-10, 2017. URL: http://www.tojet. net/volumes/v16i2.pdf\#page $=11$

[60] C. Cartagena; A. Vásquez; M. BenjumeaArias y A. Valencia-Arias. "Proposed Model for Measuring Customer Satisfaction with Telecommunications Services". Mediterranean Journal of Social Sciences, Vol. 8, No 2, pp. 15-26, 2017. DOI: 10.5901/ mjss.2017.v8n2p15.

[61] A. Valencia; O. Salazar y D. Ovalle. "Improving the Entrepreneur's Market Research Strategies Learning Process
Using the MaREMAS Environment". En: Highlights on Practical Applications of Agents and Multi-Agent Systems. Springer Berlin Heidelberg, pp. 363-374. 2013. DOI: 10.1007/978-3-642-38061-7_34.

[62] D. Gaviria; J. Arango y A. Valencia. "Reflections about the use of information and communication technologies in accounting education". Procedia-Social and Behavioral Sciences, Vol. 176, pp. 992-997, 2015. DOI: 10.1016/j.sbspro.2015.01.569.

[63] J. Arango; D. Gaviria y A. Valencia. "Differential calculus teaching through virtual learning objects in the field of management sciences". Procedia-Social and Behavioral Sciences, Vol. 176, pp. 412-418, 2015. DOI: 10.1016/j.sbspro.2015.01.490.

[64] J. Cuadros; J. Valencia y A. Valencia. "Las tecnologías de la información y la comunicación en entornos de aprendizaje rural como mecanismos de inclusión social". Actualidades Pedagógicas. Vol. 60, pp. 101-120, 2012. DOI: http://dx.doi.org/10.19052/ap.1756.

[65] V. Rodríguez-Lora; M. Henao-Cálad y A. Valencia Arias. "Taxonomies of techniques and tools for Knowledge Engineering: guide for knowledge project development". Ingeniare. Revista chilena de ingeniería, Vol. 24, N $^{\circ} 2$, pp. 351-360, 2016. DOI: 10.4067/S0718-33052016000200016.

[66] S. Chalela; A. Valencia, J. Bermúdez y C. Ortega. "Percepciones estudiantiles acerca del uso de nuevas tecnologías en instituciones de Educación Superior en Medellín”. Revista Lasallista de Investigación, Vol. 13, Nº 2, 2016.

[67] P. Rivera; P. Sánchez; E. Romo; A. Jaramillo y A. Valencia. "Percepciones de los estudiantes universitarios frente al aprendizaje por medio de dispositivos móviles". Revista Educación y Desarrollo Social. Vol. 7, No 2, 152-165, 2013. URL: https://revistas.unimilitar.edu. co/index.php/reds/article/view/687/444.

[68] M. Velez; D. Gutiérrez y A. Valencia. "Student's Perception and Expectation towards the Creation and Implementation of a Technology Management Laboratory at the Metropolitan Technology Institute MTI". Periodica Polytechnica. Social and Management Sciences, Vol. 23. № 2, pp. 98105, 2015. URL: https://pp.bme.hu/so/article/ viewFile/7915/6786. 
[69] A. Valencia; M. Benjumea y V. RodríguezLora. "Intención de uso del e-learning en el programa de Administración Tecnológica desde la perspectiva del modelo de aceptación tecnológica". Revista Electrónica Educare, Vol. 18. $\mathrm{N}^{\mathrm{o}}$ 2, pp. 247-264, 2014. DOI: 10.15359/ree.18-2.13.

[70] A. Valencia, S. Chalela, J. Bermúdez, and L. Bedoya, "Individual Factors that Encourage the Use of Virtual Platforms of Administrative Sciences Students: A Case Study". TOJET: The Turkish Online Journal of Educational Technology. Vol. 14, $\mathrm{N}^{\mathrm{o}} 3$, pp. 81-87, 2015. URL: http://www.tojet. net/articles/v14i3/1438.pdf

[71] A. Valencia, G. González, and M. Castañeda, "Structural equation model for studying the mobile-learning acceptance". IEEE Latin America Transactions. Vol. 14. No 4, pp. 1988-1992, 2016. DOI: 10.1109/ TLA.2016.7483544.
[72]. L. Bran; K. Romero; L. Echeverri; J. Peña; S. Vásquez; M. Aguilera; C. Herazo y A. Valencia. "Information and Communication Technologies Influence on Family Relationship". Global Journal of Health Science. Vol. 9, No 6, pp. 204213, 2017. DOI:10.5539/gjhs.v9n6p204.

[73] A. Valencia-Arias; D. Morales-Zapata; L. Vanegas-Rendón y M. Benjumea-Arias. "Percepción y conocimiento de los docentes universitarios sobre los procesos investigativos universitarios: estudio de caso". Educação e Pesquisa. Febrero, 2017. DOI: 10.1590/ s1517-9702201702150010.

[74] J. Bermudez; S. Chalela; J. Valencia y A. Valencia. "Research Trends in the Study of ICT Based Learning Communities: A Bibliometric Analysis". EURASIA Journal of Mathematics, Science \& Technology Education. Vol. $13 \mathrm{~N}^{\mathrm{o}} 5$, pp. 1539-1562. 2017. DOI: 10.12973/ eurasia.2017.00684a. 